\title{
Efficient routing on scale-free networks based on local information
}

\author{
Chuan-Yang Yin, Bing-Hong Wang* Wen-Xu Wang, Tao Zhou, and Hui-Jie Yang \\ Department of Modern Physics and Nonlinear Science Center, \\ University of Science and Technology of China, Hefei, 230026, PR China
}

(Dated: November 13, 2018)

\begin{abstract}
In this letter, we propose a new routing strategy with a single free parameter $\alpha$ only based on local information of network topology. In order to maximize the packets handling capacity of underlying structure that can be measured by the critical point of continuous phase transition from free flow to congestion, the optimal value of $\alpha$ is sought out. By investigating the distributions of queue length on each node in free state, we give an explanation why the delivering capacity of the network can be enhanced by choosing the optimal $\alpha$. Furthermore, dynamic properties right after the critical point are also studied. Interestingly, it is found that although the system enters the congestion state, it still possesses partial delivering capability which do not depend on $\alpha$. This phenomenon suggests that the capacity of the network can be enhanced by increasing the forwarding ability of small important nodes which bear severe congestion.
\end{abstract}

PACS numbers: 89.75.Hc, 89.20.Hh, 05.10.-a, 05.65.-b, 89.75.-k, 05.70.Ln

Since the seminal work on the small-world phenomenon by Watts and Strogatz [1] and scale-free networks by Barabási and Albert [2], the evolution mechanism of the structure and the dynamics on the networks have recently generated a lot of interests among physics community [3, 4]. One of the ultimate goals of the current studies on complex networks is to understand and explain the workings of systems built upon them [5, 6, 7, 8, 9, 10], and relatively, how the dynamics affect the network topology 11, 12, 13, 14]. We focus on the traffic dynamics upon complex networks, which can be applied everywhere, especially the vehicle flow problem on networks of roads and the information flow dynamic on interconnection computer networks. Some previous works have focused on finding the optimal strategies for searching target on the scale-free networks [15] and others have investigated the dynamics of information flow with respect to the packets handling capacity of the communication networks 16, 17, 18, 19, 20, 21, 22], however, few of which incorporate these two parts. In this letter, we address a new routing strategy based on the local information in order to both minimize the packets delivering time and maximize the capacity of huge communication networks.

In order to obtained the shortest path between any pair of nodes, one has to know the whole network structure completely. However, due to the huge size of the modern communication networks and continuous growth and variance of the networks' structure, it is usually an impossible task. Even though the network is invariant, for the sake of routing packet along the shortest path each node has to put all the shortest paths between any pair of nodes into its routing table, which is also impractical for huge size because of limited storage capacity. Therefore, In contrast to previous works allowing the data pack-

*Electronic address: bhwang@ustc.edu.cn

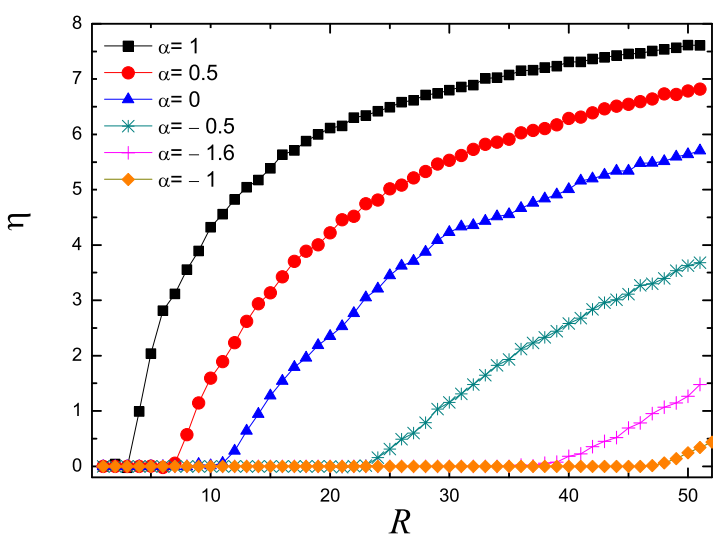

FIG. 1: (color online). The order parameter $\eta$ versus $R$ for BA network with different free parameter $\alpha$.

ets forwarding along the shortest path, in our model, we assume each node only has the topology knowledge of it's neighbors. For simplicity, we treat all nodes as both hosts and routers for generating and delivering packets. The node capacity, that is the number of data packets a node can forward to other nodes each time step, is also assumed to be a constant for simplicity. In this letter, we set $C=10$.

Recent studies indicate that many communication networks such as Internet and WWW are not homogeneous like random and regular networks, but heterogeneous with degree distribution following the power-law distribution $P(k) \sim k^{-\gamma}$. Barabási and Albert proposed a simple and famous model (BA for short) called scale-free networks [2] of which the degree distribution are in good accordance with real observation of communication networks. Here we use BA model with $m=5$ and network size $N=1000$ fixed for simulation. Our model is de- 


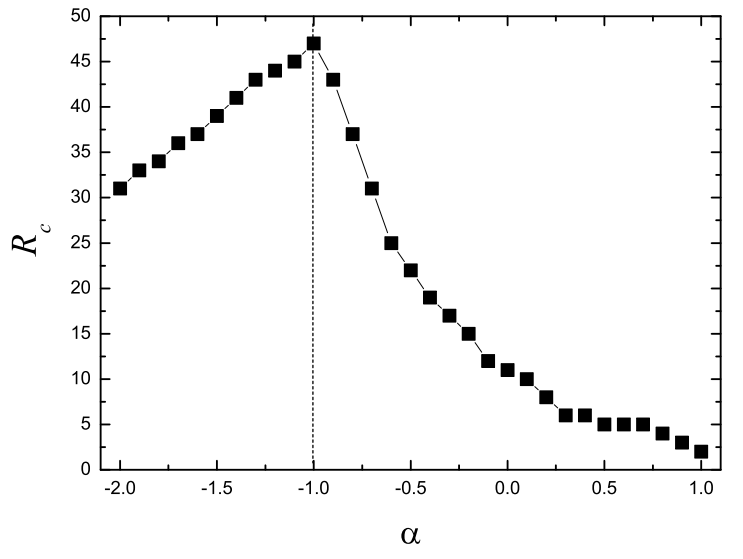

FIG. 2: The critical $R_{c}$ versus $\alpha$. The maximum of $R_{c}$ corresponds to $\alpha=-1$ marked by dot line.

scribed as follows: at each time step, there are $R$ packets generated in the system, with randomly chosen sources and destinations, and all nodes can deliver at most $C$ packets toward their destinations. To navigate packets, each node performs a local search among its neighbors. If the packet's destination is found within the searched area, it is delivered directly to its target, otherwise, it is forwarded to a neighbor node according to the preferential probability of each node:

$$
\Pi_{i}=\frac{k_{i}^{\alpha}}{\sum_{j} k_{j}^{\alpha}},
$$

where the sum runs over the neighbors of node $i$ and $\alpha$ is an adjustable parameter. Once the packet arrives at its destination, it will be removed from the system. We should also note that the queue length of each node is assumed to be unlimited and the FIFO (first in first out) discipline is applied at each queue [20]. Another important rule called path iteration avoidance (PIA) is that a path between a pair of nodes can not be visited more than twice by the same packet. Without this rule the capacity of the network is very low due to many times of unnecessary visiting of the same links by the same packets, which does not exist in the real traffic systems.

One of the most interesting properties of traffic system is the packets handling and delivering capacity of the whole network. As a remark, it is different between the capacity of network and nodes. The capacity of each node is set to be constant, otherwise the capacity of the entire network is measured by the critical generating rate $R_{c}$ at which a continuous phase transition will occur from free state to congestion. The free state refers to the balance between created packets and removed packets at the same time. While if the system enters the jam state, it means the continuous packets accumulating in the system and finally few packets can reach their destinations. In order

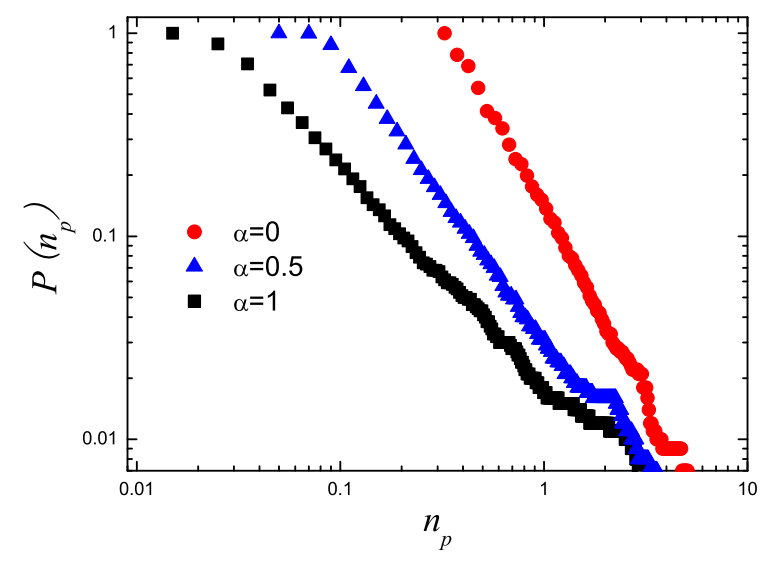

FIG. 3: The queue length cumulative distribution on each node by choosing different $\alpha$ more than zero. Data are consistent with power-law behavior.

to describe the critical point accurately, we use the order parameter 17]:

$$
\eta(R)=\lim _{t \rightarrow \infty} \frac{C}{R} \frac{\left\langle\Delta N_{p}\right\rangle}{\Delta t},
$$

where $\Delta N_{p}=N(t+\Delta t)-N(t)$ with $\langle\cdots\rangle$ indicates average over time windows of width $\Delta t$ and $N_{p}(t)$ represents the number of data packets within the networks at time $t$. For $R<R_{c},\langle\Delta N\rangle=0$ and $\eta=0$, indicating that the system is in the free state with no traffic congestion. Otherwise for $R>R_{c}, \eta \rightarrow \infty$, the system will collapse ultimately. As shown in Fig. 1, the order parameter versus generating rate $R$ by choosing different value of parameter $\alpha$ is reported. It is easy to find that the capacity of the system is not the same with variance of $\alpha$, thus, a natural question is addressed: what is the optimal value of $\alpha$ for maximizing the network's capacity? Simulation results demonstrate that the optimal performance of the system corresponds to $\alpha \approx-1$. Compared to previous work by Kim et al. [15], one of the best strategies is PRF corresponding to our strategy with $\alpha=1$. By adopting this strategy a packet can reach its target node most rapidly without considering the capacity of the network. This result may be very useful for search engine such as google, but for traffic systems the factor of traffic jam can not be neglected. Actually, average time of the packets spending on the network can also be reflected by system capacity. It will indeed reduce the network's capacity if packets spend much time before arriving at their destinations. Therefore, choosing the optimal value of $\alpha=-1$ can not only maximize the capacity of the system but also minimize the average delivering time of packets in our model.

To better understand why $\alpha=-1$ is the optimal choice, we also investigate the distribution of queue length on each node with different $\alpha$ in the stable state. 


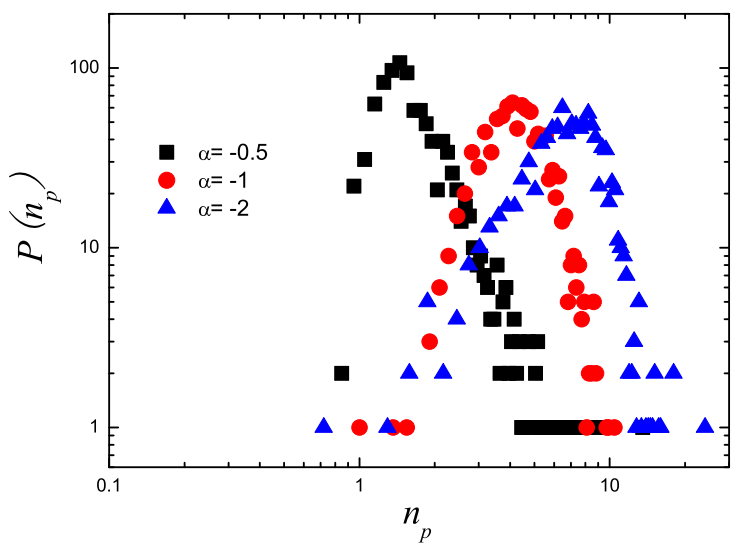

FIG. 4: The queue length cumulative distribution on each node by choosing different $\alpha$ less than zero. $P\left(n_{p}\right)$ approximately exhibits a Poisson distribution.

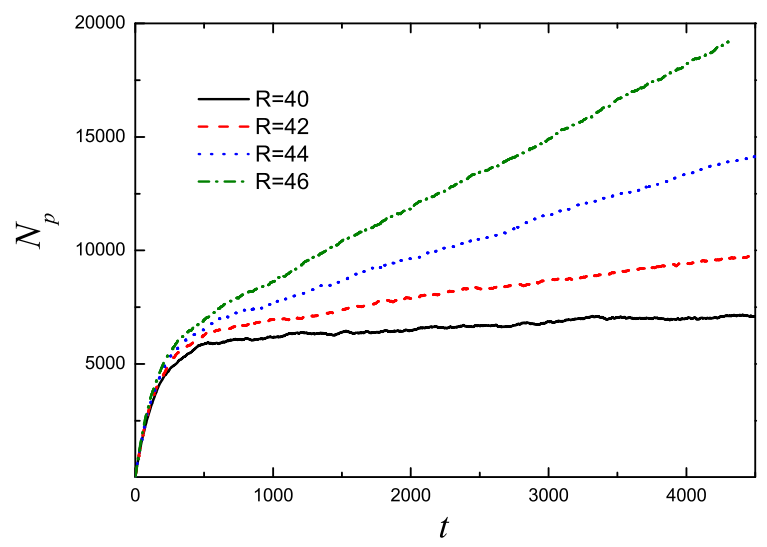

FIG. 5: The evolution of $N_{p}$ for $R>R_{c}$. Here, $\alpha_{c}$ takes -1.5 corresponding to the critical point $R_{c}=39$.

Fig. 3 shows that when $\alpha \geq 0$, the queue length of the network follows the power-law distribution which indicates the highly heterogenous traffic on each node. Some nodes with large degree bear severe traffic congestion while few packets pass through the others and the heterogenous behavior is more obviously correspondent to the slope reduction with $\alpha$ increase from zero. But due to the same delivering capacity of all nodes, this phenomenon will undoubtedly do harm to the system because of the severe overburden of small numbers of nodes. In contrast to Fig. 3, Fig. 4 shows better condition of the networks with queue length approximately displays the Poisson distribution which represents the homogenous of each node like the degree distribution of random graph. From this aspect, we find that the capacity of the system
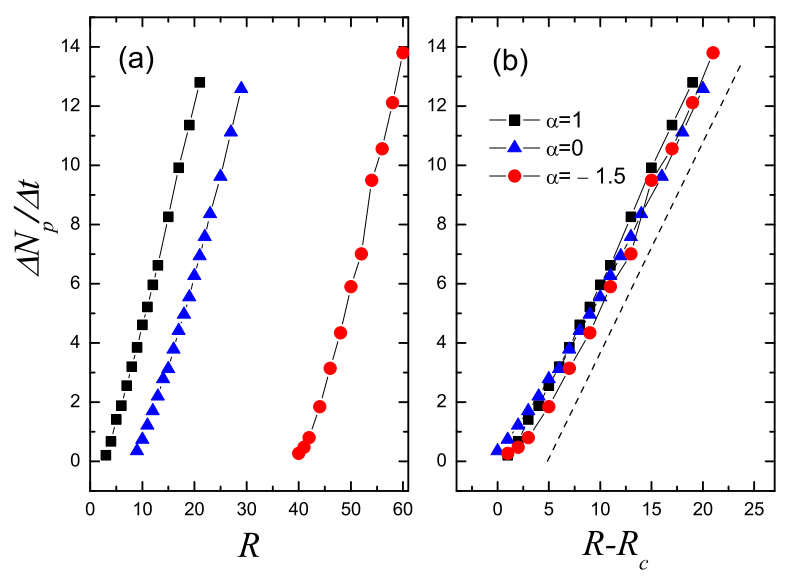

FIG. 6: The ratio between $\Delta N_{p}$ and time step interval $\Delta t$ versus $R$ (a) and versus $R-R_{c}$ the rescaling of $R$ (b) for different $\alpha$. In (b) three curves collapse to a single line with the slope $\approx 0.7$ marked by a dashed line.

with $\alpha<0$ is larger than that with $\alpha>0$. But it's not the whole story, in fact, the system's capacity is not only determined by the capacity of each node, but also by the actual path length of each packet from its source to destination. Supposing that if all packets bypass the large degree nodes, it will also cause the inefficient routing for ignoring the important effect of hub nodes on scale-free networks. By the competition of these two factors, the nontrivial value $\alpha=-1$ is obtained with the maximal network's capacity.

The behavior in jam state is also interesting for alleviating traffic congestion. Fig. 5 displays the evolution of $N_{p}(t)$ i.e. the number of packets within the network with distinct $R$. $\alpha$ is fixed to be -1.5 and $R_{c}$ for $\alpha=-1.5$ is 39. All the curves in this figure can be approximately separated into two ranges. The starting section shows the superposition of all curves which can be explained by the fact that few packets reach their destinations in a short time so that the increasing velocity of $N_{p}$ is equal to $R$. Then after transient time, $N_{p}$ turns to be a linear function of $t$. Contrary to our intuition, the slope of each line is not $R-R_{c}$. We investigate the increasing speed of $N_{p}$ with variance of $R$ by choosing different parameter $\alpha$. In Fig. 6(a), in the congestion state $N_{p}$ increases linearly with the increment of $R$. Surprisingly, after $x$ axis is rescaled to be $R-R_{c}$, three curves approximately collapse to a single line with the slope $\approx 0.7$ in Fig. $6(\mathrm{~b})$. On one hand, this result indicates that in the jam state and $R$ is not so large, the dynamics of the system do not depend on $\alpha$. On the other hand the slope less than 1 reveals that not all the $R-R_{c}$ packets are accumulated per step in the network, but about 30 percent packets do not pass through any congested nodes, thus they can reach their destination without contribution to the net- 


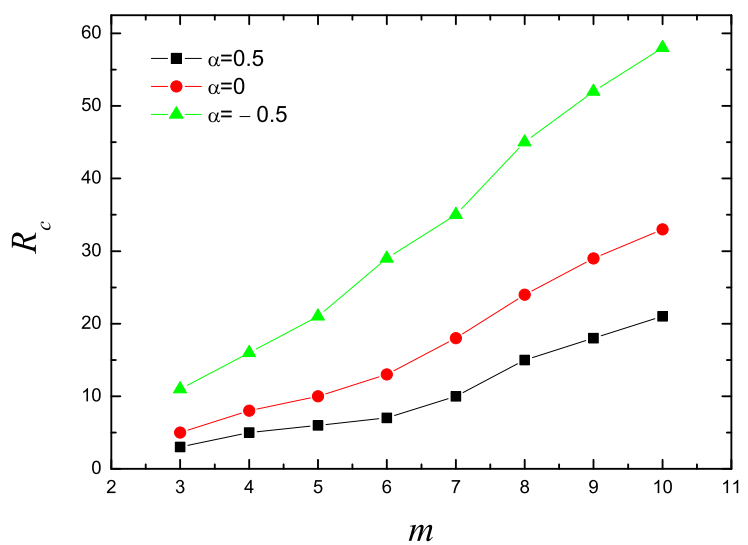

FIG. 7: The variance of $R_{c}$ with the increasing of $m$.

work congestion. This point also shows that when $R$ is not too large in the congestion state, the congested nodes in the network only take the minority, while most other nodes can still work. Therefore, the congestion of the system can be alleviated just by enhancing the processing capacity of a small number of heavily congested nodes. Furthermore, we study the variance of critical point $R_{c}$ affected by the link density of BA network. As shown in Fig. 7, increasing of $m$ obviously enhances the capac- ity of BA network measured by $R_{c}$ due to the fact that with high link density, packets can more easily find their target nodes.

Motivated by the problem of traffic congestion in large communication networks, we have introduced a new routing strategy only based on local information. Influenced by two factors of each node's capacity and navigation efficiency of packets, the optimal parameter $\alpha=-1$ is obtained with maximizing the whole system's capacity. Dynamic behavior such as increasing velocity of $N_{p}$ in the jam state shows the universal properties which do not depend on $\alpha$. In addition, the property that scale-free network with occurrence of congestion still possesses partial delivering ability suggests that only improving processing ability of the minority of heavily congested nodes can obviously enhance the capacity of the system. The variance of critical value $R_{c}$ with the increasing of $m$ is also discussed. Our study may be useful for designing communication protocols for large scale-free communication networks due to the local information the strategy only based on and the simplicity for application. The results of current work also shed some light on alleviating the congestion of modern technological networks.

The authors wish to thank Na-Fang Chu, Gan Yan, $\mathrm{Bo} \mathrm{Hu}$ and Yan-Bo Xie for their valuable comments and suggestions. This work is funded by NNSFC under Grants No. 10472116, 70271070 and 70471033, and by the Specialized Research Fund for the Doctoral Program of Higher Education (SRFDP No.20020358009).
[1] Watts D J and Strogatz S H 1998 Nature 393440

[2] Barabási A L and Albert R 1999 Science 286509

[3] Albert R and Barabási A L 2002 Rev. Mod. Phys. 7447

[4] Dorogovtsev S N and Mendes J F F 2002 Adv. Phys. 51 1079

[5] Pastor-Satorras R and Vespignani A 2001 Phys. Rev, Lett. 863200

[6] Yan G, Zhou T, Wang J, Fu Z Q and Wang B H 2005 Chin. Phys. Lett. 22510

[7] Zhou T, Yan G and Wang B H 2005 Phys. Rev. E 71 046141

[8] Goh K I, Lee D S, Kahng B and Kim D 2003 Phys. Rev. Lett. 91148701

[9] Zhou T and Wang B H 2005 Chin. Phys. Lett. 221072

[10] Yang H J et al 2004 Phys. Rev. E 69066104

[11] Wang W X, Wang B H, Hu B et al. 2005 Phys. Rev. Letts. 94188702

[12] Wang W X, Hu B, Wang B H and Yan G 2005 preprint
arXiv:cond-mat/0505419

[13] Wang W X, Hu B, Zhou $\mathrm{T}$ et al 2005 preprint arXiv:cond-mat/0504062

[14] Zhu C P et al 2004 Phys. Rev. Lett. 92218702

[15] Kim B J et al 2002 Phys. Rev. E 65027103

[16] Holme P and Kim B J 2002 Phys. Rev. E 65066109

[17] Arenas A, Díaz-Guilera A and Guimerà R 2001 Phys. Rev. Letts 863196

[18] Guimerà R, Díaz-Guilera A, Vega-Redondo F, Cabrales A and Arenas A 2002 Phys. Rev. Letts 89248701

[19] Tadić B, Thurner S and Rodgers G J 2004 phys. Rev. E 69036102

[20] Zhao L, Lai Y C, Park K and Ye N 2005 Phys. Rev. E 71026125

[21] Singh B K and Gupte N 2005 Phys. Rev. E 71055103

[22] Yan G, Zhou T, Hu B, Fu Z Q and Wang B H 2005 preprint arXiv:cond-mat/0505366 